\title{
Validación de la versión en español del "Cuestionario de Autoevaluación del Control de la Vejiga (CACV). Un nuevo instrumento para detectar pacientes con disfunción del tracto urinario inferior
}

\author{
Espuña Pons $\mathrm{M}^{*}$, Puig Clota $\mathrm{M}^{*}$, Rebollo Álvarez $\mathrm{P}^{* *}$. \\ *Institut Clínic de Ginecología, Obstetricia i Neonatología. Hospital Clínic. Universidad de Barcelona. \\ ${ }^{* *}$ BAP Health Outcomes. Oviedo.
}

Actas Urol Esp. 2006;30(10):1017-1024

\section{RESUMEN}

VALIDACIÓN DE LA VERSIÓN EN ESPAÑOL DEL “CUESTIONARIO DE AUTOEVALUACIÓN DEL CONTROL DE LA VEJIGA” (CACV). UN NUEVO INSTRUMENTO PARA DETECTAR PACIENTES CON DISFUNCIÓN DEL TRACTO URINARIO INFERIOR

Introducción. Teniendo en cuenta la elevada prevalencia del síndrome de Vejiga Hiperactiva-VH ("urgencia”, con o sin incontinencia, a menudo asociada a aumento de frecuencia y/o nocturia) en nuestro país y la dificultad de identificarlo, sería interesante tener un instrumento en forma de cuestionario corto autocontestado para detectar y evaluar los pacientes con sintomas del tracto urinario inferior. El objetivo del estudio fue evaluar las propiedades psicométricas de la versión española del Cuestionario de Autoevaluación del Control de la Vejiga (CACV).

Pacientes y método. Se incluyeron consecutivamente a 133 mujeres que consultaron por síntomas urinarios en una unidad especializada, para la realización de un estudio urodinámico. Las pacientes cumplimentaron el CACV (escalas "síntomas" y "molestias") y el ICIQ-UI SF y fueron sometidas al protocolo habitual: historia uroginecológica, exploración pélvica y estudio urodinámico. Se calcularon factibilidad, validez y fiabilidad del CACV, así como la curva COR para la puntuación de las dos escalas del CACV respecto al diagnóstico urodinámico de Hiperactividad del Detrusor (HD).

Resultados. Factibilidad: tiempo medio de administración, 3,5(1,5) minutos; 2 mujeres no contestaron ninguno de los cuestionarios. Validez: las puntuaciones de "sintomas" y "molestias" fueron superiores en las mujeres diagnosticadas de HD: "sintomas" $=7,8(2,6)$ vs $6,2(2,3)(\mathrm{p}=0,0002)$; “molestias" $=9,1(3)$ vs $7,8(3,6)(\mathrm{p}=0,03)$; el coeficiente de correlación de Spearman entre la escala "molestia" y el ítem 3 del ICIQ-UI SF (afectación) fue de 0,65(p<0,001). Fiabilidad: el alfa de Cronbach para "síntomas" fue 0,722 y para "molestias" 0,889. Según la curva COR, para ambas escalas, la puntuación igual o superior a 6 puntos mostró adecuada sensibilidad y especificidad en el diagnóstico de HD.

Conclusiones: El Cuestionario de Autoevaluación del Control de la Vejiga-CACV es un instrumento de sencillo uso que muestra adecuadas factibilidad, validez y fiabilidad para ser empleado en la práctica clínica como instrumento de cribado para VH.

Palabras clave: Vejiga Hiperactiva. Incontinencia de orina. Mujer. Diagnóstico.

\begin{abstract}
VALIDATION OF THE SPANISH VERSION OF THE “BLADDER CONTROL SELF-ASSESSMENT QUESTIONNARIE” (B-SAQ). A NEW SCREENING INSTRUMENT FOR LOWER URINARY TRACT DYSFUNCTION

Background: Taking into account the high prevalence in our country of Overactive Bladder Syndrome (OAB) ("urgency", with or without incontinence, sometimes associated to an increase in frequency and/or nocturia) and the difficulty to identify it, it would be interesting to have a short patient self assessment screening questionnarie for the evaluation of lower urinary tract symptoms. The objective of the study is to evaluate the psychometric properties of the Spanish version of the Bladder control Self-Assessment Questionnaire (B-SAQ).

Patients and methods: 133 women consulting due to lower urinary tract symptoms in a health care centre specialised on uroginecology. The subjects filled the B-SAQ (scales "symptoms" and "discomfort") and the ICIQ-UI SF, and underwent the usual protocol: uroginecologic history, pelvic examination and urodynamic study. Feasibility, validity, and reliability of the B-SAQ were assessed, as well as the ROC curve for the scores of both BCSEQ scales regarding the urodynamic diagnosis of Detrusor Overactivity (DO).

Results: Feasibility: Average administration time, 3.5 (1.5) minutes; 2 women did not answer any of the questionnaires. Validity: the scores for "symptoms" and "discomfort" were higher in those women with a DO diagnosis: "symptoms" = 7.8 (2.6) vs 6.2 (2.3) ( $\mathrm{p=0.0002);} \mathrm{"discomfort"} \mathrm{=} 9.1$ (3) vs 7.8 (3.6) ( $\mathrm{p}=0.03)$; the Spearman's correlation coefficient between "discomfort" scale and item 3 of the ICIQ-UI SF (affection) was 0.65 ( $\mathrm{p}<0.001$ ). Feasibility: Cronbach's alpha for "symptoms" was 0.722 and 0,889 for "discomfort". According to the COR curve for both scales, a score greater than or equal to 6 showed adequate sensibility and specificity for the DO diagnosis.

Conclusions: The B-SAQ is an easy-use instrument which shows adequate feasibility, validity and reliability for its use in clinical practice as screening instrument for $\mathrm{OAB}$.
\end{abstract}

Keywords: Overactive Bladder. Urinary Incontinence. Female. Diagnosis. 
$\mathrm{E}^{1}$ "Síndrome de Vejiga Hiperactiva (VH)" se define como la "urgencia", con o sin incontinencia de urgencia, a menudo asociada a aumento de frecuencia y/o nocturia. También se denomina "síndrome de urgencia" o "síndrome de urgencia-frecuencia" ${ }^{1}$. Los citados términos pueden usarse si no hay infección probada u otra patología orgánica demostrable que pueda causar sintomatología similar. Estas combinaciones de sintomas son sugestivas de hiperactividad del músculo detrusor demostrable por urodinámica. La VH incluye hiperactividad del músculo detrusor de origen neurogénico (insuficiente inhibición cortical, neuropatías degenerativas y lesiones medulares) y no neurogénico (envejecimiento, obstrucción de salida e irritación crónica de la vejiga por tumores o ITU), así como hipersensibilidad del músculo ${ }^{2}$. Los datos epidemiológicos sobre la VH son muy escasos. En un estudio $^{3}$ se entrevistaron a 16.776 personas de edad igual o superior a 40 años de 6 países, entre ellos España. La prevalencia de Vejiga Hiperactiva evaluada en el conjunto de estos países Europeos (Francia, Alemania, Italia, España, Suecia y Reino Unido) resultó ser sorprendentemente alta en sujetos de 40 ó más años: 16,6\%. El síntoma Frecuencia (85\%) fue el más frecuente, seguido de urgencia (54\%) e IU de Urgencia (36\%). La prevalencia de los sintomas de $\mathrm{VH}$ aumentaba con la edad. El 60\% de los sujetos del estudio con síntomas habían consultado con el médico, pero sólo el $27 \%$ estaban recibiendo actualmente tratamiento. La prevalencia en EE.UU. es similar a la encontrada en Europa, de acuerdo a los datos del estudio NOBLE (National Overactive Bladder Evaluation) con una cifra muy parecida para varones y mujeres (16 y $16,9 \%$ respectivamente) ${ }^{4}$. En un estudio recientemente publica$\mathrm{do}^{5}$, se estudió la prevalencia de VH en España con una muestra de 1.669 sujetos de la población general (50,6\% mujeres). En este estudio se determinó una prevalencia de $25,6 \%$ en mujeres mayores de 40 años. Sin embargo, hay que señalar que estos estudios se realizaron sobre datos de los síntomas de $\mathrm{VH}$ de los pacientes obtenidos por entrevista, telefónica en la mayoría de los casos o en persona, y no a través de la autocumplimentación de un cues- tionario específico como recomienda la tercera International Consultation on Incontinence ${ }^{6}$. Existen algunos cuestionarios específicamente diseñados para detectar la $\mathrm{VH}$ que han sido adecuadamente validados, pero no en nuestro país. Entre ellos el más conocido probablemente sea el Overactive Bladder Symptom and Health Related Quality of Life $(O A B-Q)^{7}$ que ha demostrado ser psicométricamente robusto en su versión original, pero que no ha sido utilizado en muestras amplias de la población y que además tiene 33 ítems en su versión corta (62 en la versión completa) lo que dificulta su uso en la práctica clínica. Recientemente se ha publicado la validación del Patient Perception of Bladder Condition $(P P B C)^{8}$ al que los autores presentan como una medida global de auto-evaluación sobre el estado de la vejiga en pacientes con VH. Sin embargo los propios autores recomiendan usar otros instrumentos de medida junto con este cuestionario en la evaluación del resultado del tratamiento de la VH.

El impacto que el síndrome de $\mathrm{VH}$ produce en quien lo padece es importante a juzgar por los resultados publicados, interfiriendo en aspectos del funcionamiento físico, social y sexual, así como en el sueño, el trabajo y las relaciones personales ${ }^{9-11}$. En un estudio realizado en 11.521 sujetos de 6 países de Europa, entre ellos España, ${ }^{12}$ se comprobó que el $32 \%$ de los que padecían $\mathrm{VH}$ afirmaron que los síntomas le hacían sentir deprimido y el $28 \%$ que se sentían muy estresados. Además sufrian impacto en su trabajo y actividades diarias por temer las interrupciones causadas por los síntomas de VH.

Teniendo en cuenta la elevada prevalencia de mujeres que padecen el síndrome de $\mathrm{VH}$ en nuestro país y el considerable porcentaje de ellas que no son fácilmente identificables, tener un instrumento en forma de cuestionario corto autocontestado para detectar y evaluar los pacientes con síntomas del tracto urinario inferior. El objetivo del presente estudio fue estudiar las propiedades psicométricas (factibilidad, validez y fiabilidad) de la versión española del Cuestionario de Autoevaluación del Control de la Vejiga (CACV) para poder recomendar su uso en la práctica clínica habitual. 


\section{PACIENTES Y MÉTODO}

Se incluyeron consecutivamente a 133 mujeres que cumplían con el único criterio de inclusión de acudir a la consulta de la Unidad de Uroginecologia del ICGON del Hospital Clinic de Barcelona por síntomas urinarios, para estudio urodinámico. Se equilibró la muestra incluyendo a 30 mujeres con sintomatología de IU con predominio de urgencia, 30 con predominio de IU de esfuerzo y 30 con sintomatología mixta; durante el tiempo de recogida de datos de estas pacientes se incluyeron en el estudio a otras 43 mujeres con otros síntomas relacionados sugestivos de VH. Las mujeres incluídas no cumplían ninguno de los siguientes criterios de exclusión:

1. Los propios del estudio urodinámico: infección urinaria, hematuria, dolor vesical asociado sin infección.

2. Enfermedad neurológica diagnosticada.

3. Prolapso genital mayor de grado II (anterior, posterior o central).

4. Incapacidad para entender los mensajes y órdenes precisos para llevar a cabo el procedimiento del estudio urodinámico o la respuesta al cuestionario.

Después de recabar de la paciente el consentimiento para participar en el estudio todas las pacientes fueron sometidas al protocolo diagnóstico habitual en la Unidad de Uroginecología que consta de historia uroginecológica, exploración pélvica y estudio urodinámico. Previamente a la realización de estas pruebas cumplimentaron el "Cuestionario de Autoevaluación del Control de la Vejiga" que se pretendía validar. Si contestaban afirmativamente a la pregunta 4 de la escala "síntomas" que hace referencia a si tienen incontinencia urinaria (IU), se les pedía que cumplimentasen además la versión española del ICIQ-UI SF, cuestionario específico para la evaluación de la IU.

El cuestionario "Cuestionario de Autoevaluación del Control de la Vejiga" (CACV) fue diseñado por un grupo europeo de expertos en disfunciones del tracto urinario inferior, con el objetivo de que a través de la autocumplimentación del cuestionario las personas con $\mathrm{VH}$ se identifiquen como que padecen este problema y puedan solicitar ayuda médica o bien puedan ser detectadas en la consulta médica mediante este instrumento. Este sencillo cuestionario de tan sólo 8 ítems agrupados en dos escalas ("molestias" y "sintomas") fue desarrollado siguiendo la metodología habitual que incluye la realización de un panel de pacientes y otro de expertos que permitieron reducir el número de preguntas a las 8 de la versión final. El cuestionario fue desarrollado originalmente en inglés. Para obtener la versión española utilizada en el presente estudio se realizó una adaptación cultural incluyendo un doble proceso de traducción - retrotraducción realizado por dos personas bilingües, siendo la versión final en Español aprobada por el equipo investigador.

El ICIQ-UI SF es un cuestionario que está orientado a la detección de la IU en cualquier ámbito asistencial. La versión final del cuestionario, que se ha traducido y adaptado culturalmente en diversos países, consta de 3 items ("Frecuencia”, "Cantidad" y "Afectación"), más un grupo de 8 preguntas relacionadas con el tipo de IU que no forman parte de la puntuación del cuestionario, y que tienen únicamente finalidad descriptiva y orientadora sobre el tipo de incontinencia de orina. La puntuación total, resultado de la suma de los tres primeros ítems, oscila entre 0 y 21 puntos. La versión española de este cuestionario ha demostrado tener adecuadas propiedades psicométricas ${ }^{13,14}$.

En una hoja de datos estandarizada se recogieron los siguientes datos de cada paciente: edad, paridad, menopausia (si/no), tratamiento hormonal sustitutivo (si/no), peso y talla, variables del examen físico, los datos del estudio urodinámico y las respuestas a los cuestionarios.

Respecto al estudio urodinámico se recogieron los parámetros urodinámicos cuantitativos siguientes: volumen a primera sensación; volumen a primer deseo miccional; volumen a deseo miccional fuerte; volumen a capacidad cistométrica máxima; presión del detrusor a capacidad máxima; acomodación vesical; presión del detrusor al inicio de la micción; presión del detrusor máxima durante la micción; y residuo postmiccional (postcistometría).

De este modo se dispuso de una prueba subjetiva de evaluación de la Vejiga Hiperactiva, la puntuación en la escala "síntomas" del Cuestionario de Autoevaluación del Control de la Vejiga que se pretendia validar, y otra objetiva que sirvió de referencia, la prueba urodinámica. También para la puntuación en la escala de 
"molestia" del Cuestionario de Autoevaluación del Control de la Vejiga se dispuso de una referencia con la que estudiar su validez: la escala de "afectación" del Cuestionario ICIQ-UI SF.

\section{ANÁLISIS ESTADÍSTICO}

1. Evaluación de la Factibilidad del cuestionario de Autoevaluación del Control de la Vejiga: se realizó en función de la respuesta a los ítems del cuestionario, evaluando el tanto por ciento de pacientes que no respondió a cada uno de los ítems.

2. Evaluación de la Validez del cuestionario de Autoevaluación del Control de la Vejiga: se evaluó la validez de criterio comparando la puntuación en la escala "síntomas" del cuestionario con el diagnóstico urodinámico de Hiperactividad del detrusor (HD). Esto se hizo analizando la diferencia en la puntuación total de la escala "síntomas" entre los pacientes que padecen y no padecen HD de acuerdo al diagnóstico urodinámico. La hipótesis de la que se partía era que la puntuación de "síntomas" sería superior en el grupo de pacientes con diagnóstico urodinámico de HD. Por otro lado, se estudió la correlación entre la escala "molestia" del cuestionario a examen y la escala de afectación del ICIQ-UI SF.

3. Evaluación de la Fiabilidad del cuestionario de Autoevaluación del Control de la Vejiga: se evaluó en términos de consistencia interna mediante el cálculo del coeficiente alfa de Cronbach. Se planteaba como hipótesis previa el obtener un coeficiente igual o mayor a 0,7 considerado por separado para las dos escalas: "sintomas" y "molestia".

Para determinar el punto de corte en la puntuación que permitiese identificar a los pacientes con una aceptable sensibilidad, se calculó la curva COR para la puntuación de ambas escalas del cuestionario ("síntomas" y "molestia") respecto al diagnóstico urodinámico de Hiperactividad del Detrusor ya fuera ésta pura o mixta.

\section{RESULTADOS}

Se estudiaron a 133 mujeres con una edad media de 57,5 años (D.E.= 15,5), un IMC medio de $29,4(18,5)$ y una paridad media de 2,4 partos $(1,6)$. La mayoría de las mujeres eran menopáusicas $(69,9 \%)$ siendo la edad media de inicio de la misma 48,5 años $(4,9)$ y sólo $8(6 \%)$ recibían tratamiento hormonal sustitutivo.
Respecto a la exploración física, 57 mujeres $(42,9 \%)$ demostraron IU de Esfuerzo y 71 mujeres $(53,4 \%)$ hipermovilidad uretral; 32 (24,1\%) presentaban prolapso uterino, que era grado I en 13 mujeres (9,8\%) y grado II en 19 (14,3\%); 60 $(45,1 \%)$ presentaban cistocele, que era grado I en 24 mujeres (18\%) y grado II en $36(27,1 \%)$; y 30 mujeres $(22,6 \%)$ presentaban rectocele, que era grado I en 16 mujeres (12\%) y grado II en 14 $(10,6 \%)$.

Las puntuaciones medias de las pacientes estudiadas en los cuestionarios CACV e ICIQ-UI SF se presentan en la Tabla 1.

Tabla 1

Cuestionario de Autoevaluación del Control de la Vejiga (CACV) y Cuestionario ICIQ-IU-SF. Resultados de las puntuaciones medias de los cuestionarios en las pacientes estudiadas

Puntuaciones del cuestionario CACV $(n=131)$

\begin{tabular}{lc} 
CACV-1 (Sintoma: URGENCIA ) & $1,9(0,9)$ \\
CACV-2 (Sintoma: FRECUENCIA ) & $1,9(0,8)$ \\
CACV-3 (Sintoma: NOCTURIA) & $1,6(0,9)$ \\
CACV-4 (Sintoma: INCONTINENCIA) & $1,6(0,9)$ \\
CACV-Sintomas & $\mathbf{6 , 9}(\mathbf{2 , 6 )}$ \\
CACV-5 (Molestia: URGENCIA ) & $2,2(0,9)$ \\
CACV-6 (Molestia: FRECUENCIA ) & $2,1(1)$ \\
CACV-7 (Molestia: NOCTURIA ) & $1,9(1)$ \\
CACV-8 (Molestia: INCONTINENCIA ) & $2,1(0,9)$ \\
CACV-Molestia & $\mathbf{8 , 3}(\mathbf{3 , 4})$ \\
\hline \multicolumn{1}{c}{$\quad$ Puntuación del cuestionario ICIQ-UI SF } \\
$\quad \quad$ (mujeres con sintomas de IU) (n=113) \\
ICIQ-1 (Frecuencia) & $3,6(1,3)$ \\
ICIQ-2 (Cantidad) & $3,7(1,5)$ \\
ICIQ-3 (Afectación) & $7,1(3)$ \\
ICIQ-Total & $\mathbf{1 4 , 4}(\mathbf{4 , 6 )}$
\end{tabular}

Los resultados sobre los parámetros urodinámicos se presentan en la Tabla 2. De acuerdo a la prueba urodinámica resultaron diagnosticadas de IU de esfuerzo (IUE) 40 pacientes $(30,1 \%)$; de Hiperactividad del Detrusor (HD), 51 (38,4\%); de IU mixta, 24 (18\%); de disfunción del vaciado, 4 (3\%); en 10 mujeres $(7,5 \%)$ el estudio urodinámico fue normal, es decir no se evidenció ninguna alteración funcional que justificara la sintomatología y no se dispuso del resultado de la prueba urodinámica en 4 casos (3\%). 
Tabla 2

Resultados de parámetros urodinámicos cuantitativos en las pacientes estudiadas distribuidas según el estudio urodinámico evidenciara hiperactividad del detrusor (HD) o una incontinencia de esfuerzo pura (IUE)

\begin{tabular}{lcc}
\hline & HD (+) & IUE (+) \\
\hline Vol. a primera sensación $(\mathrm{n}=83)$ & $173,85(84,88)$ & $195,64(82,55)$ \\
Vol. a primer deseo miccional $(\mathrm{n}=131)$ & $213,70(92,01)$ & $248,56(81,33)$ \\
Vol. a deseo miccional fuerte $(\mathrm{n}=131)$ & $308,29(107,71)$ & $364,80(95,01)$ \\
Vol. a capacidad cistométrica máxima $(\mathrm{n}=131)$ & $418,11(130,27)$ & $505,77(143,57)$ \\
Presión detrusor a capacidad máxima $(\mathrm{n}=116)$ & $35,46(17,45)$ & $27,70(16,15)$ \\
Acomodación vesical (n=122) & $41,11(56,83)$ & $91,29(75,18)$ \\
Presión detrusor al inicio de micción (n=115) & $24,21(12,65)$ & $15,61(8,73)$ \\
Presión detrusor máxima durante micción $(\mathrm{n}=119)$ & $26,05(12,41)$ & $27,49(53,11)$ \\
Residuo postmiccional (n=118) & $43,87(63,60)$ & $19,52(37,34)$
\end{tabular}

HD-Hiperactividad del detrusor/IUE-Incontinencia Urinaria de Esfuerzo

1. Evaluación de la Factibilidad del cuestionario de Autoevaluación del Control de la Vejiga (CACV).

El tiempo medio (D.E.) de administración del cuestionario fue de 3,5 $(1,5)$ minutos. De las 133 mujeres incluidas en el estudio tan sólo 2 no contestaron ninguno de los ítems del cuestionario y tampoco cumplimentaron los items del ICIQ-UI SF.

\section{Evaluación de la Validez del cuestionario CACV.}

La puntuación de las escalas de síntomas y molestias del CACV en el grupo de pacientes con diagnóstico urodinámico de HD fueron superiores a las del grupo de pacientes con diagnósticos urodinámicos diferentes de HD (Tabla 3). Esto no ocurrió para otros diagnósticos urodinámicos como el de IUE (Tabla 3). En esta misma tabla puede verse que también, de acuerdo a los síntomas evaluados por el cuestionario ICIQ-UI $\mathrm{SF}$, las mujeres que expresan síntoma de urgencia tienen puntuación superior en la escala síntomas que las que no lo manifiestan. Por otro lado, el coeficiente de correlación de Spearman entre la escala de molestia

\section{Tabla 3}

Puntuaciones del Cuestionario de Autoevaluación del Control de la Vejiga (CACV) en las pacientes estudiadas distribuidas según el diagnóstico urodinámico de hiperactividad del detrusor (HD) o de incontinencia urinaria de esfuerzo (IUE) y según refieran síntomas de IU de urgencia o de IU de esfuerzo en el cuestionario ICIQ-UI-SF

\begin{tabular}{|c|c|c|c|}
\hline \multicolumn{4}{|c|}{ Puntuaciones del cuestionario CACV según Dco. Urodinámico $(n=128)$} \\
\hline & HD (urodinámica + ) & HD (urodinámica - ) & $\mathbf{p}$ \\
\hline CACV-Sintomas & $7,8(2,6)$ & $6,2(2,3)$ & 0,0002 \\
\hline \multirow[t]{2}{*}{ CACV-Molestia } & $9,1(3)$ & $7,8(3,6)$ & 0,033 \\
\hline & IUE (urodinámica +) & IUE (urodinámica - ) & $\mathbf{p}$ \\
\hline CACV-Síntomas & $6,7(2,3)$ & $7,3(2,8)$ & 0,26 \\
\hline CACV-Molestia & $8,3(3,3)$ & $8,6(3,4)$ & 0,56 \\
\hline \multicolumn{4}{|c|}{ Puntuación del cuestionario CACV según sintomas del ICIQ-UI SF ( $n=114)$} \\
\hline & Sint. Urgencia (ICIQ +) & Sint. Urgencia (ICIQ - ) & $\mathbf{p}$ \\
\hline CACV-Síntomas & $7,9(2,5)$ & $6,6(2,4)$ & 0,008 \\
\hline \multirow[t]{2}{*}{ CACV-Molestia } & $9,3(3,1)$ & $8,4(3,2)$ & 0,118 \\
\hline & Sint. Esfuerzo (ICIQ +) & Sint. Esfuerzo (ICIQ - ) & $\mathbf{p}$ \\
\hline CACV-Síntomas & $7,1(2,4)$ & $7,6(2,8)$ & 0,334 \\
\hline CACV-Molestia & $8,9(3,2)$ & $8,6(3,2)$ & 0,729 \\
\hline
\end{tabular}




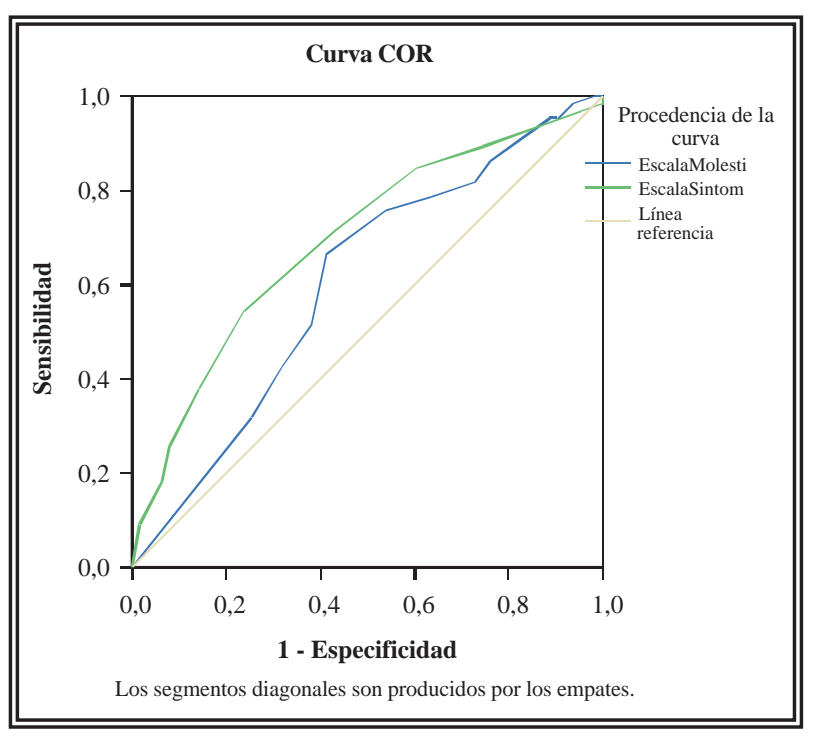

FIGURA 1. Curvas COR calculada para las escalas "sintomas" y "molestia" del cuestionario CACV respecto al diagnóstico urodinámico de HD"

$84,8 \%$ y una especificidad del 39,7\%; la misma puntuación en la escala "molestias" mostró una sensibilidad del 81,8\% y una especificidad del 27\%.

Utilizando este punto de corte para la escala “síntomas" del CACV, 94 mujeres $(70,7 \%)$ resultaron diagnosticadas de $\mathrm{VH}$. La mayoría de estas mujeres $(n=89)$ respondieron afirmativamente al item 4 del cuestionario CACV sobre pérdidas de orina; utilizando el cuestionario ICIQ-UI SF para el diagnóstico clínico de la IU entre este grupo de mujeres, se comprueba que 88 son clasificadas correctamente por el CACV como incontinentes y 5 como continentes, quedando tan sólo una mujer que el cuestionario $\mathrm{CACV}$ califica de incontinente, pero el ICIQ-UI SF no (sensibilidad=100\%; especificidad=83\%). Respecto al diagnóstico urodinámico de HD (pura o mixta) el cuestionario CACV califica correctamente a 55 como VH/HD y 4 sin VH/HD; por otro lado califica a 34 mujeres como $\mathrm{VH}$ y por tanto sugestivas de tener HD, que no pudo ser demostrada en el estudio urodinámico realizado y finalmente se observó que 1 mujer con $\mathrm{HD}$ en la urodinámica no había sido detectada con el CACV (sensibilidad=98,1\%; especificidad=10,5\%).

\section{DISCUSIÓN}

El Cuestionario de Autoevaluación del Control de la Vejiga-CACV es un instrumento de sencillo uso que muestra adecuadas factibilidad, validez y fiabilidad para ser empleado en la práctica clínica como instrumento de cribado para VH. La brevedad de su extensión (tan sólo 8 ítems) y la sencillez de su puntuación e interpretación son características que lo hacen útil para la detección de la IU en cualquier ámbito asistencial, así como en estudios epidemiológicos.

La principal limitación del presente trabajo es que la muestra estudiada incluye sólo mujeres y todas atendidas en un servicio especializado de uroginecología, incluyendo un escaso número de controles sanos (10 mujeres con prueba urodinámica de normalidad). Son necesarios estudios poblacionales que incluyan varones y sujetos de ambos sexos sin problemas de incontinencia.

La principal dificultad que se presenta en la validación de escalas de síntomas, como lo es el cuestionario CACV, estriba en la difícil comparación con un "patrón oro" que facilite el análisis de validez. En el presente estudio al tratarse de mujeres que consultaban en una unidad especializada en IU, la mayoría de ellas mostraban incontinencia además de los síntomas de VH, lo cual permitió el análisis de validez respecto al diagnóstico urodinámico de $\mathrm{HD}$, a sabiendas de que son necesarios estudios que incluyan una muestra más heterogénea respecto a los síntomas y a la presencia o no de IU. De todos modos es de esperar que en un estudio más amplio se confirmen los resultados del presente, dado que en general se considera que es más difícil para una escala de síntomas el discriminar entre grupos de pacientes dentro de un diagnóstico genérico (IU) que entre pacientes y sanos.

La factibilidad del cuestionario CACV, de acuerdo a los resultados presentados, es buena. El corto tiempo de administración lo convierte en una herramienta útil para ser utilizada en la detección de $\mathrm{VH}$ en una consulta médica del primer nivel asistencial.

Respecto a la validez de criterio, las pacientes con diagnóstico urodinámico de hiperactividad del detrusor (HD) mostraron puntuaciones superiores en la escala "síntomas" a las del resto de mujeres estudiadas, tal y como se había hipotetizado. También la escala "molestias" mostró adecuada validez a través del moderado coeficiente de correlación que mostró tener con la escala de afectación del cuestionario ICIQ-UI SF previa- 
mente validado ${ }^{13,14}$. Un dato más a favor de la validez del cuestionario $\mathrm{CACV}$ es la elevada sensibilidad que mostró tener la puntuación en el item 4 de las pacientes con 6 o más puntos en la escala "síntomas" frente al diagnóstico de IU (por la puntuación del ICIQ-UI SF y por la prueba urodinámica).

Por último los datos sobre consistencia interna de las dos escalas del cuestionario, medida a través del coeficiente alfa de Cronbach, fueron también satisfactorios y confirmaron las hipótesis planteadas al inicio del estudio (alfa $>0,7$ ).

El cálculo de la curva COR permitió determinar un punto de corte para la puntuación en ambas escalas que permitiese utilizar el cuestionario para aquello para lo que fue diseñado: detectar pacientes con $\mathrm{VH}$ en un primer nivel asistencial. El punto de corte propuesto (6 puntos) tiene un valor balanceado de sensibilidad (S) y 1-especificidad (1-E) que permite recomendar su uso en la detección de pacientes con $\mathrm{VH}$ : escala "síntomas" $\mathrm{S}=84,8 \%$ y $1-\mathrm{E}=60,3 \%$; escala "molestias" $\mathrm{S}=81,8 \%$ y 1 $\mathrm{E}=73 \%$.

Aunque la sensibilidad al cambio no ha sido evaluada en el presente trabajo, la elevada fiabilidad, medida por el alfa de Cronbach, indica que probablemente el cuestionario $\mathrm{CACV}$ es útil en el seguimiento de pacientes antes y después de la aplicación de tratamientos para la $\mathrm{VH}$.

El cuestionario CACV se presenta como una alternativa buena al empleo del OAB- ${ }^{7}$, que tiene 33 items en su versión corta y por tanto es difícilmente aplicable en la práctica clínica y al empleo del $\mathrm{PBBC}^{8}$, que precisa de otros instrumentos para la evaluación del resultado de los tratamientos. El presente cuestionario resultará sin duda muy útil tanto en la práctica clínica, para evaluar al paciente en un primer nivel asisten- cial, como en la investigación, permitiendo la realización de estudios epidemiológicos de prevalencia de enfermedad evaluada desde el punto de vista de la paciente que es esencial de acuerdo a las recomendaciones de la tercera International Consultation on Incontinence ${ }^{6}$ (Fig. 2).

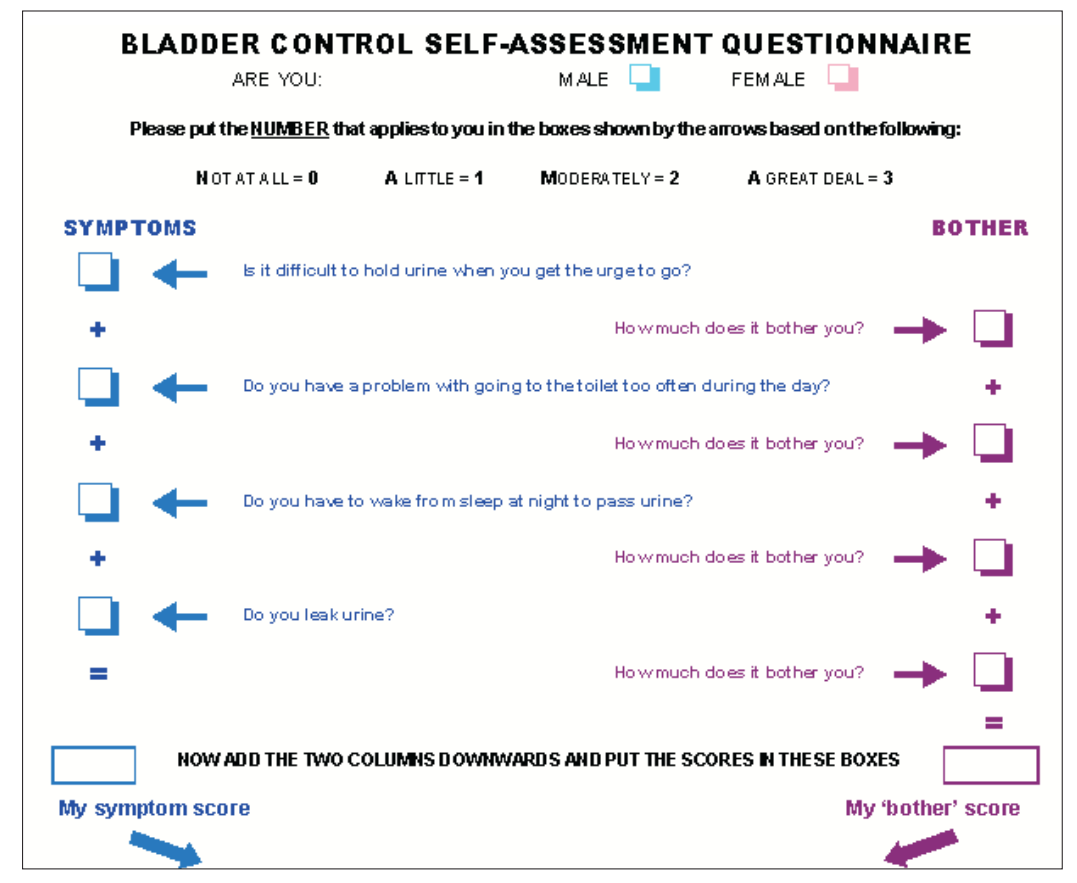

CUESTIONARIO DE AUTOEVALUACIÓN DEL CONTROL DE LA VEJIGA (Spanish version of the Bladder Control Self-Assessment Questionnaire) ES USTED: $\quad$ HOMBRE $\square \quad$ MUJER $\square$

Por favor, anote el NÚMERO aplicable a su caso en las casillas indicadas por las flechas, teniendo en cuenta lo siguiente:

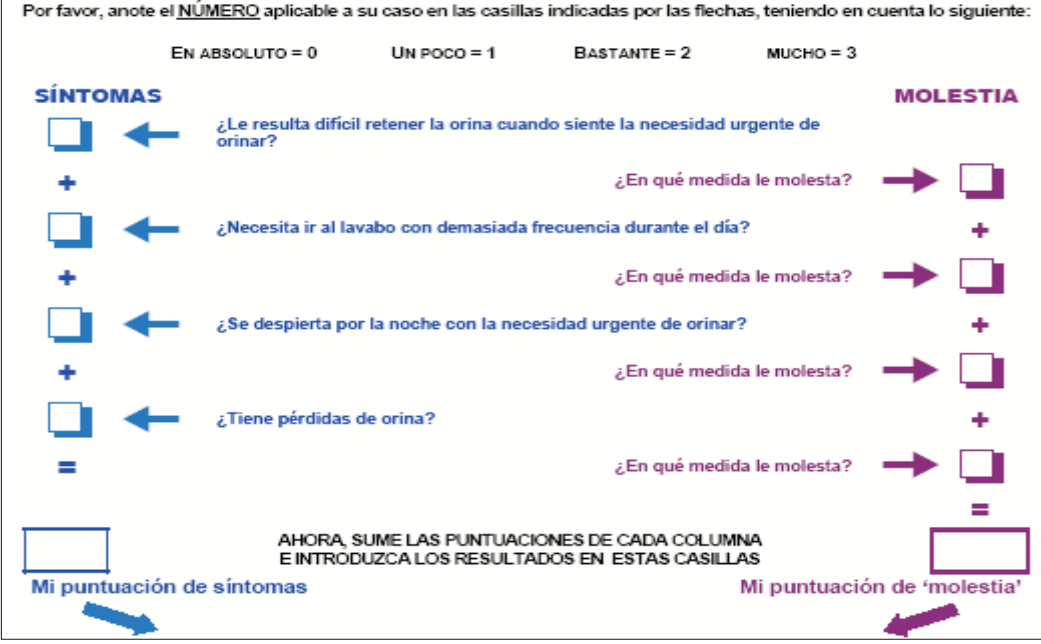

FIGURA 2. Cuestionario para detectar mujeres con vejiga hiperactiva. Versión original en inglés "Bladder Control self-assessment Questionnaire" (B-SAQ) y versión traducida y validada al español "Cuestionario de Autoevaluación del control de la vejiga" (CACV). Este cuestionario ha sido diseñado por: Karl-Erik Andersson, Linda Cardozo, David Castro-Diaz, Chris Chapple, Dirk de Ridder, Montserrat Espuna Pons, Markus Hohenfellner, Francois Haab, Con Kelleher, Mike Kirby, Ian Milsom, Prof PE Van Kerrebroeck, Dr ME Vierhout, Adrian Wagg. 


\section{Agradecimientos}

Agradecemos a Maite González, enfermera de investigación, becada para colaborar en la realización de este estudio, su entusiasmo y dedicación para poder llevar a cabo este proyecto. A las enfermeras Amelia Pérez, Lidia Jover y Maria José Martínez, de la Unidad de Suelo Pélvico del Institut Clínic de Ginecología, Obstetricia y Neonatología, por su colaboración en el reclutamiento de las pacientes.

Para la realización de este estudio se solicitó una beca a ASTELLAS PHARMA, S.A. que permitió financiar la recogida y análisis de los datos.

\section{REFERENCIAS}

1. Abrams P, Cardozo L, Khoury S, Wein A. Incontinence. 3rd International Consultation on Urinary Incontinence. Edition 2005. Plymbridge, Mass: Health Publication Ltd. United Kingdom; 485-517.

2. Hampel C, Gillitzer R, Pahernik S, Hohenfellner M, Thuroff JW. Epidemiology and etiology of overactive bladder. Urologe A. 2003;42(6):776-786.

3. Milsom I, Abrams P, Cardozo L, Roberts RG, Thuroff J, Wein AJ. How widespread are the symptoms of an overactive bladder and how are they managed? A population-based prevalence study. BJU Int. 2001;87(9):760-766..

4. Stewart WF, Van Rooyen JB, Cundiff GW, Abrams P, Herzog AR, Corey R, Hunt TL, Wein AJ. Prevalence and burden of overactive bladder in the United States World J Urol. 2003 May;20(6):327-336.

5. Castro D, Espuña M, Prieto M, Badía X. Prevalencia de vejiga hiperactiva en España: estudio poblacional. Arch Esp Urol. 2005;58(2):131-138.

6. Abrams P, Cardozo L, Khoury S, Wein A. Incontinence. 3rd International Consultation on Urinary Incontinence. Edition 2005. Plymbridge, Mass: Health Publication Ltd. United Kingdom: 521.

7. Coyne K, Revicki D, Hunt T, Corey R, Stewart W, Bentkover J, Kurth H, Abrams P. Psychometric validation of an overactive bladder symptom and health-related quality of life questionnaire: the OAB-q. Qual Life Res. 2002;11(6):563-574.
8. Coyne KS, Matza LS, Kopp Z, Abrams P. The validation of the patient perception of bladder condition (PPBC): a single-item global measure for patients with overactive bladder. Eur Urol 2006; 49(6):1079-1086.

9. Stewart WF, Van Rooyen JB, Cundiff GW, Abrams P, Herzog AR, Corey R, Hunt TL, Wein AJ. Prevalence and burden of overactive bladder in the United States. World $\mathrm{J}$ Urol 2003; 20(6):327-336.

10. Coyne KS, Zhou Z, Bhattacharyya SK, Thompson CL, Dhawan R, Versi E. The prevalence of nocturia and its effect on health-related quality of life and sleep in a community sample in the USA. BJU Int. 2003;92(9):948-954.

11. Coyne KS, Payne C, Bhattacharyya SK, Revicki DA, Thompson C, Corey R, Hunt TL. The impact of urinary urgency and frequency on health-related quality of life in overactive bladder: results from a national community survey. Value Health. 2004;7(4):455-63.

12. Irwin DE, Milsom I, Koop Z, Abrams P, Cardozo L. Impact of overactive bladder symptoms on employment, social interactions and emotional well-being in six European countries. BJU Int 2006;97(1):96-100.

13. Espuña P, Rebollo P, Puig M. Validación de la versión española del ICIQ-SF. Un cuestionario para evaluar la incontinencia urinaria. Med Clin (Barc) 2004;122:288-292.

14. Espuña Pons M, Puig Clota M, Rebollo P, Vanrell Díaz JA, Iglesias Guiu X. Evaluación del resultado del tratamiento quirúrgico de la incontinencia urinaria de esfuerzo femenina mediante el cuestionario ICIQ-UI SF. Med Clin (Barcelona) 2005; 124:772-774.

Dra. M. Espuña Pons

E-mail: 12922mep@comb.es

(Trabajo recibido el 26 de junio 2006) 